\title{
Characterization Associated with the Frequent Severe Exacerbator Phenotype in COPD Patients
}

\author{
Yao-Kuang $\mathrm{Wu}^{1,2}$ \\ Wen-Lin Su ${ }^{1,2}$ \\ Mei-Chen Yang ${ }^{1,2}$ \\ Sin-Yi Chen' \\ Chih-Wei Wu' \\ Chou-Chin Lan ${ }^{1,2}$ \\ 'Division of Pulmonary Medicine, \\ Department of Internal Medicine, Taipei \\ Tzu Chi Hospital, Buddhist Tzu Chi \\ Medical Foundation, New Taipei City, \\ Taiwan; ${ }^{2}$ School of Medicine, Tzu-Chi \\ University, Hualien, Taiwan
}

\begin{abstract}
Background: Chronic obstructive pulmonary disease (COPD) is a chronic inflammatory disease with exacerbations causing hospitalizations, morbidity, and mortality. COPD exacerbation causes a substantial health impact, and its subtypes might differ in prognosis and treatment response.

Methods: This study evaluated the factors of COPD exacerbations and explored the probabilities of frequent severe COPD exacerbations. Categorical and continuous variables between groups were compared. The hazard ratio (HR) and the probability of no hospital readmission were also estimated.
\end{abstract}

Results: A total of 617 COPD patients were enrolled and comprised the frequent exacerbator $(\mathrm{N}=226)$ and the non-frequent exacerbator $(\mathrm{N}=391)$ groups. The frequent exacerbator group significantly displayed a higher eosinophil count $(\mathrm{EC} ; \mathrm{p}=0.004)$, a higher percentage of the frequent severe acute exacerbation history before the index hospitalization (IH; $p<0.001)$, a lower FEV1 value ( $\mathrm{p}=0.001)$, and a higher triple combination inhaler prior and following the $\mathrm{IH}(\mathrm{p}<0.001$ and $\mathrm{p}=0.002)$ than the non-frequent exacerbator one. Increasing age (aOR of 1.02), higher EC (aOR of 1.09), and lower FEV1 value (aOR of 0.72 ) were significantly associated with an increased hospital readmission risk. The readmission rate and risk were higher in patients with a history of frequent severe acute exacerbation (aHR of 3.38) than those without severe acute exacerbation. Cases treated with the triple combination inhaler before the IH had a higher readmission rate and risk than non-users.

Conclusion: Patients with EC $\geq 2 \%$, FEV1 $<50 \%$, or frequent severe acute exacerbation history before the IH have a higher risk of being diagnosed with a frequent exacerbator phenotype. Besides, higher age, triple combination inhaler before the $\mathrm{IH}$, and smoking might be independently correlated with the frequent readmission risk within 1-year postexacerbation. A better comprehension of the COPD exacerbation mechanism may further identify the best course of preventative strategy and lead to novel interventions.

Keywords: chronic obstructive pulmonary disease, COPD, forced expiratory volume in one second, FEV1, forced vital capacity, FVC, index hospitalization, eosinophil count

\section{Introduction}

Chronic obstructive pulmonary disease (COPD) is a chronic inflammation disease of the airways characterized by an incompletely reversible airflow limitation. ${ }^{1}$ The COPD disease progression is punctuated by periodic deterioration in respiratory symptoms called exacerbations that cause most hospitalizations followed by significant morbidity and mortality. ${ }^{2}$ COPD exacerbations could lead to hospital admissions contributing to a significant clinical and economic load worldwide. ${ }^{3}$ Exacerbations might get more frequent and more severe as COPD progresses, ${ }^{4}$ and
Correspondence: Chou-Chin Lan Division of Pulmonary Medicine, Department of Internal Medicine, Taipei Tzu Chi Hospital, Buddhist Tzu Chi Medical Foundation, No. 289, Jianguo Road, Xindian District, New Taipei City, Taiwan

Tel +886-2-66289779 ext 5709

Fax +886-2-66289009

Email bluescopy@yahoo.com.tw 
their recurrence is correlated with a lung function decline and health status of patients. ${ }^{5}$ The hospitalization cost of severe COPD exacerbation can be 60 times more expensive than mild or moderate exacerbations managed by primary care services. ${ }^{6}$

For patients surviving after a COPD exacerbationrelated hospitalization, readmission is a significant problem. Identifying and reducing risk factors for frequent readmission is therefore essential. ${ }^{7}$ The official American Thoracic Society workshop report on reducing COPD readmission emphasized the requirement to identify risk factors for COPD readmissions. ${ }^{8}$

Acute exacerbations are an essential feature in COPD's natural history, and identifying the frequent exacerbator phenotype may benefit from avoiding these complications. Furthermore, identifying COPD patients with risk factors associated with severe exacerbations may alert physicians, induce closer follow-up, and adopt preventive measures. Identification of biomarkers enables phenotype-driven approaches to manage and prevent exacerbations.

COPD exacerbations are heterogeneous, and various phenotypes that differ in biological basis, prognosis, and response to therapy have been proposed. ${ }^{9}$ The specific phenotype of frequent exacerbations in COPD patients has been identified using different thresholds primarily derived from the median exacerbation frequency in various cohorts, containing two or more moderate-to-severe exacerbations during 1 year. ${ }^{9}$ Consequently, most researchers use this cutoff to describe the frequent exacerbator phenotype, ${ }^{4,10}$ and this definition is now recorded in the Global Initiative for Chronic Obstructive Lung Disease guidelines (GOLD). ${ }^{1}$

This study's primary aim was to develop and validate possible predictors of frequent severe exacerbations in COPD patients requiring hospitalization (two or more exacerbations per year) in reliance on data collection of the clinical records obtained during hospital admission. The secondary aim was to investigate the independent clinical parameters involved in frequent readmission risk during 1 year after an exacerbation.

\section{Materials and Methods Study Design}

An observational and retrospective cohort study was conducted in a tertiary education hospital, which screened the medical records for COPD-related hospitalizations from January 2016 to December 2019. The inclusion criteria: 1)
Patients admitted to the hospital from outpatient or emergency department with a severe acute exacerbation. 2) Patients who were previously diagnosed with COPD by a specialist pulmonologist and assigned one of the ICD 10 (International Classification of Diseases) codes, J44, J44.0, J44.1, J44.8, and J44.9. 3) Patients with no exacerbation in one month before preceding enrollment. 4) Patients with more than one diagnosis code of J44 and sub-segment. 5) Patients having COPD-related hospital admission for at least one time during this period. The definition of index hospitalization (IH) was the first admission related to COPD during the study period, and patients were survived at the IH. This study's protocol was approved by Institutional Review Board, Taipei Tzu Chi Hospital, Buddhist Tzu Chi Medical Foundation in August 2020 (Protocol number 09-X-059). The informed consent was signed and provided by each included participant.

The COPD diagnosis was based on a spirometry and compatible history. COPD was defined as the volume of post-bronchodilator forced expiratory in one second (FEV1)/forced vital capacity (FVC) ratio $<0.7$ according to 2020 GOLD guidelines. ${ }^{1}$ Severe exacerbation of COPD was identified as more than 2 consecutive days with sustained worsening of patient's symptoms beyond day-today variations causing hospitalization. Only COPD severe exacerbations were accounted for and merged for in the analysis. $^{2}$ Frequent severe exacerbation was defined as hospital readmission within 1 year over 2 times from chest diseases outpatient or emergency department. ${ }^{3}$ Nonfrequent exacerbator was defined as patients with less than two hospital readmissions for severe acute exacerbation within 1 year after hospital discharge. The number of hospitalizations within 1 year after hospital discharge had already included the IH during the study period. During the candidate reassessment, the exclusion criteria were as follows: absence of obstructive pattern on pulmonary function test (PFT) results, absence of a valid PFT result before $\mathrm{IH}$, mislabeled as asthmatic (Non-smokers with or without obstructive spirometry results and no COPD record in medical records), tuberculosis, diagnosis result of lung cancer in the prior 3 years, those unable to complete the follow-up, and those who received systemic steroids within $48 \mathrm{~h}$ before the blood test at the IH. COPD patients with severe exacerbations were classified into two groups conforming to their frequency count 1 year after the IH: The frequent exacerbator group (Severe acute exacerbation with hospitalization $\geq 2$ ) and 


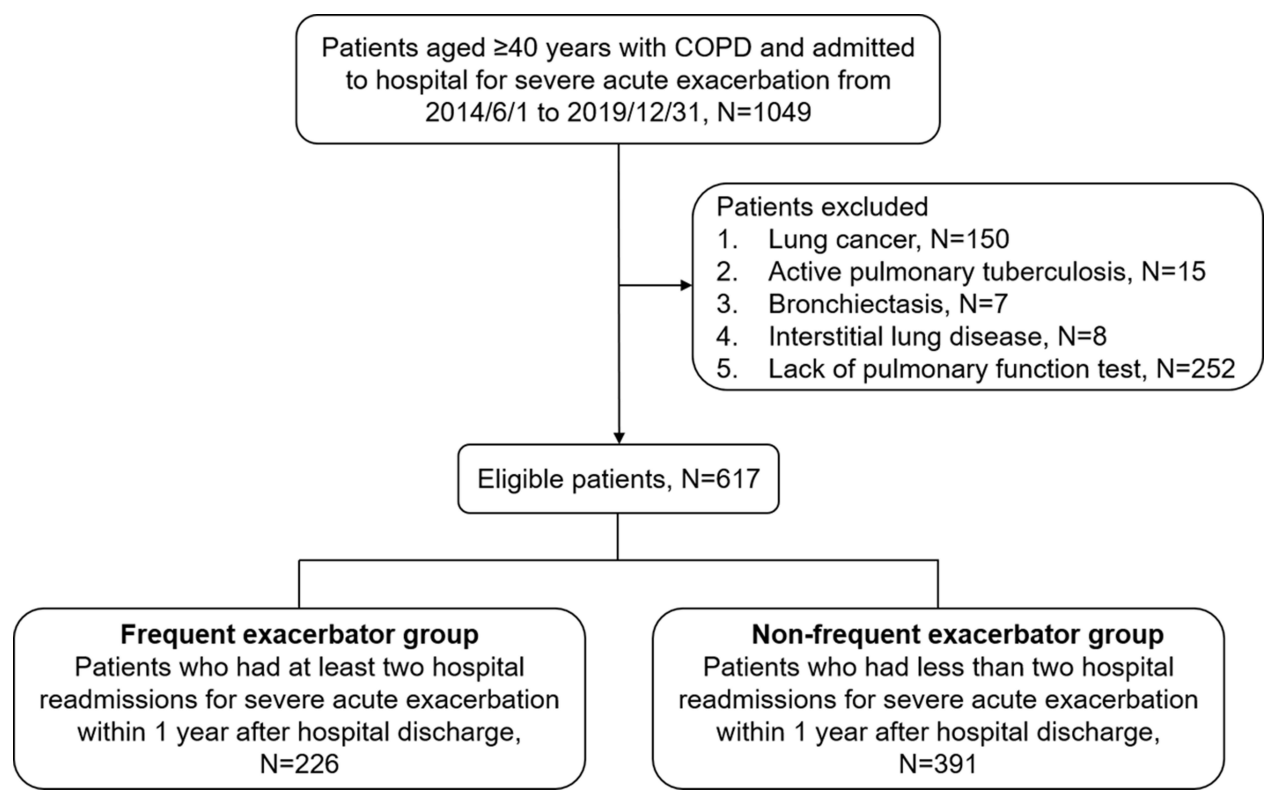

Figure I Flowchart of selected population in this study.

the non-frequent exacerbator group $(\leq 1)$. The subject distribution is summarized in Figure 1.

\section{Measurement}

The following data collected according to the medical records before the IH: demographics, body mass index, smoking history, comorbidities (the neuromuscular disease included stroke, Parkinsonism), severe exacerbations' frequency in previous 12 months, the use of home noninvasive ventilation, pulmonary function test, and the respiratory medicines including inhaled corticosteroids (ICS), long-acting beta-agonists (LABA), and long-acting antimuscarinic agents (LAMA). The following data were collected during the $\mathrm{IH}$ : arterial blood gas, C-reactive protein (CRP) levels, chest X-ray (CXR), and hemogram measured during a corticosteroid-free time frame, defining as no use of systemic corticosteroid from $1 \mathrm{~h}$ to $48 \mathrm{~h}$ prior venipuncture.

Primary in-hospital treatments were recorded, including antibiotics, systemic steroids at $\mathrm{IH}$, rate of intensive care unit (ICU) or respiratory care center (RCC) utilization, and mechanical ventilation. Hospital stays and inhaler prescriptions after discharge were also recorded.

\section{Outcomes}

The primary outcome was to compare clinical characteristics, laboratory findings, and treatment between frequent exacerbator and non-frequent exacerbator and the independent factors related to frequent severe exacerbation phenotype. The secondary outcomes have assessed the relationship between independent factors and the time of the first COPD-related readmission within 12 months.

\section{Ethical Statement}

The study was approved by the Institutional Review Board, Taipei Tzu Chi Hospital, Buddhist Tzu Chi Medical Foundation in August 2020 (protocol number 09-X-059).

\section{Statistical Analyses}

Baseline characteristics were compared between groups using Chi-squared tests and independent $t$-tests to detect any differences in the categorical and continuous demographic variables. Data were displayed as a number (percentage) of categorical variables and mean $\pm \mathrm{SD}$ for continuous variables. Cox regression models were used to estimate the adjusted hazard ratio (aHR) and its 95\% confidence intervals (CIs) of the risk of hospital readmission. The assumption of proportional hazards was checked, and the probability of no hospital readmission was estimated by Kaplan-Meier method. A Log rank test was used to test the differences between groups. All $\mathrm{P}$ values were two-sided with a significance level of 0.05 . Data management and subsequent statistical analyses were conducted using SAS version 9.4 software (SAS Institute, Inc.).

\section{Result}

A total of 617 COPD patients were enrolled following the inclusion and exclusion criteria (Figure 1). Of them, 226 
patients who underwent at least two hospital readmissions for severe acute exacerbation less than 1 year after hospital discharge were grouped into the frequent exacerbator group, and 391 patients who underwent less than two hospital readmissions were grouped into the non-frequent exacerbator group (Figure 1). The baseline characteristics and laboratory data of both groups are shown in Table 1 . The frequent exacerbator group displayed a significantly higher percentage of eosinophil (2.37 \pm 3.65 vs $1.60 \pm 2.20$, $\mathrm{p}=0.004)$ on hospital admission and more than 1 severe acute exacerbation in the past year prior to the IH $(12.83 \%$ vs $2.81 \%, \mathrm{p}<0.001)$ than the non-frequent exacerbator group. In addition, the GOLD 2019 classification showed a significant difference between the frequent exacerbator and non-frequent exacerbator group $(p<0.001)$. Differences in the pulmonary function and arterial blood gas tests were found between these two groups (Table 2). FEV1 value was higher in non-frequent exacerbator group ( $1.20 \pm 0.47$ vs $1.07 \pm 0.46, \mathrm{p}=0.001)$ with a higher FEV1/ FVC ratio $(56.45 \pm 11.24$ vs $52.63 \pm 12.00, \mathrm{p}<0.001)$ and $\mathrm{PH}$ value $(7.38 \pm 0.06$ vs $7.35 \pm 0.10, \mathrm{p}=0.046)$. Treatments on hospital admission and concurrent medications were also studied to delineate their association with hospital readmission frequency (Table 3). The percentage of antibiotic-steroid combination treatment was significantly higher in the frequent exacerbator group than in the nonfrequent exacerbator $(72.32 \%$ vs $60.10 \%, \mathrm{p}=0.005)$. Similar trends are also shown in patients who received a triple combination inhaler before the IH $(p<0.001)$ and following the $\mathrm{IH}(\mathrm{p}=0.002)$. There was no significant difference in both the infection status and the length of stay between the two groups.

Increasing age (aHR of 1.02), higher eosinophil count (EC; aHR of 1.09), and lower FEV1 value (aHR of 0.72) were significantly associated with an increased hospital readmission risk. Cases with a low percentage of predicted FEV1 value $(<50 \%)$ showed a significantly lower probability of no readmission during the 1-year follow-up time than cases with a high percentage of predicted FEV1 value ( $\geq 50 \%$ ) (Log rank test, $p=0.047$, Figure $2 A)$. The probability of no readmission was significantly lower in patients with a high percentage of eosinophil $(\geq 2 \%)$ when compared to patients with a low percentage of eosinophil $(<2 \%)(\mathrm{p}=0.031)$ (Figure $2 \mathrm{~B})$.

The readmission rate was higher in patients with more than one severe acute exacerbation in the past year $(63.90$ vs 16.29 per 100 person-years) and at risk (aHR of 3.38; $95 \%$ CI, 2.23-5.11; $<<0.001)$ than those with no history of severe acute exacerbation by more than three times. Cases treated with the triple combination inhaler before the IH also had a higher readmission rate (30.18 vs 13.31 per 100 person-years) and readmission risk (aHR of $1.83 ; 95 \% \mathrm{CI}, 1.33-2.54 ; \mathrm{p}<0.001)$ in comparison with the non-users. Besides, current smokers had a higher readmission rate (23.40 vs 15.62 per 100 person-years) and risk (aHR 1.43; 95\% CI, 1.01-2.03; $\mathrm{p}=0.044$ ) than nonsmokers (Table 4).

\section{Discussion}

In this study, a COPD subgroup with frequent exacerbator phenotype has been proposed with distinct outcomes compared to the non-frequent exacerbator one. Phenotypes of frequent exacerbators had been postulated and surveyed in previous studies. ${ }^{3}$ This COPD-related phenotype has recently been described using data from the Evaluation of COPD Longitudinally to Identify Predictive Surrogate Endpoints (ECLIPSE) study. ${ }^{4}$ The COPD exacerbations are clustered together in time rather than random events, and there is a high-risk period for recurrent exacerbations after the initial exacerbation. ${ }^{4}$ Le Rouzic et al confirmed the existence and clinical relevance of a subgroup of frequent exacerbator in COPD patients identified using a threshold of two exacerbations per year, allowing easy identification of routine care for at-risk patients before the prolonged follow-up. ${ }^{3}$

In the present study, the frequent exacerbator group significantly showed a higher peripheral blood eosinophil percentage (PBEC, 2.37\%) and a shorter time of first hospital readmission due to $\mathrm{COPD}$, similar to previous studies. ${ }^{11-13}$ Couillard et al reported that a patient with higher eosinophilia had a higher readmission risk due to COPD (OR of 3.59) and a shorter time to first readmission due to COPD (aHR of 2.74). ${ }^{12}$ Bélanger et al reported that the eosinophilic inflammation confers an increased risk of readmission due to COPD. ${ }^{11}$ One of our previous studies demonstrated a significant linear correlation between the blood eosinophil percentage and readmission numbers. ${ }^{13}$ A recent study on acute exacerbation of chronic obstructive pulmonary disease (AECOPD) also showed an increased exacerbation risk in patients with elevated blood eosinophils. ${ }^{14}$ With respiratory-related readmissions in the study being dominated by exacerbations, the results showed consistency with the COPD gene and ECLIPSE studies. In these studies, increased blood EC was reported to correlate with increased risk of recurrent exacerbations in stable COPD. ${ }^{15,16}$ These shreds of evidence could 
Table I Baseline Characteristics and Laboratory Findings on Admission Frequent Exacerbator Group vs Non-Frequent Exacerbator Group

\begin{tabular}{|c|c|c|c|}
\hline Characteristics & $\begin{array}{l}\text { Frequent Exacerbator Group } \\
(\mathrm{N}=226)\end{array}$ & $\begin{array}{l}\text { Non-Frequent Exacerbator Group } \\
(\mathrm{N}=39 \mathrm{I})\end{array}$ & $P$ value \\
\hline Male sex & $198(87.61)$ & $342(87.47)$ & 0.959 \\
\hline Age, year & $77.48 \pm 10.62$ & $75.83 \pm 11.02$ & 0.069 \\
\hline BMI, $\mathrm{kg} / \mathrm{m}^{2}$ & $23.49 \pm 4.02$ & $23.92 \pm 4.40$ & 0.224 \\
\hline \multicolumn{4}{|l|}{ Hemogram value } \\
\hline Leukocyte count, $10^{9} / \mathrm{L}$ & $|042| .6 \mid \pm 4489.07$ & $9970.10 \pm 4895.93$ & 0.256 \\
\hline Neutrophil count, \% & $74.47 \pm 11.67$ & $74.96 \pm 10.89$ & 0.599 \\
\hline Lymphocyte count, \% & $14.87 \pm 9.30$ & $15.20 \pm 9.22$ & 0.671 \\
\hline Monocyte count, \% & $6.75 \pm 3.52$ & $6.95 \pm 2.86$ & 0.482 \\
\hline Eosinophil count, \% & $2.37 \pm 3.65$ & $1.60 \pm 2.20$ & 0.004 \\
\hline Basophil count, \% & $0.24 \pm 0.34$ & $0.25 \pm 0.27$ & 0.843 \\
\hline Hemoglobin, g/dL & $|3.04 \pm 2.1|$ & $13.35 \pm 1.81$ & 0.061 \\
\hline Hematocrit, \% & $38.75 \pm 6.91$ & $39.56 \pm 5.87$ & 0.139 \\
\hline Platelet count, $10^{3} \mu \mathrm{L}$ & $2 \mid 4.97 \pm 77.67$ & $205.0 \mathrm{I} \pm 88.03$ & 0.146 \\
\hline Mean platelet volume, $\mathrm{fL}$ & $9.76 \pm 0.99$ & $9.90 \pm 0.80$ & 0.065 \\
\hline Neutrophil count, $10^{9} / \mathrm{L}$ & $7934.44 \pm 4032.47$ & $7692.05 \pm 4391.16$ & 0.497 \\
\hline Lymphocyte count, $10^{9} / \mathrm{L}$ & $1398.78 \pm 975.42$ & $|330.48 \pm 80| .34$ & 0.373 \\
\hline Eosinophil count, $10^{9} / \mathrm{L}$ & $218.18 \pm 365.46$ & $139.32 \pm 191.45$ & 0.003 \\
\hline Neutrophil-to-lymphocyte ratio & $9.61 \pm 12.98$ & $8.7 I \pm 10.54$ & 0.381 \\
\hline \multicolumn{4}{|l|}{ Inflammatory marker } \\
\hline CRP & $4.62 \pm 6.40$ & $4.8 I \pm 6.30$ & 0.759 \\
\hline Home noninvasive ventilation & $21(9.29)$ & $27(6.91)$ & 0.286 \\
\hline Smoking status & & & 0.313 \\
\hline Never-smoker & $84(37.17)$ & $168(42.97)$ & \\
\hline Current smoker & $59(26.11)$ & $86(21.99)$ & \\
\hline Ex-smoker & $83(36.73)$ & $137(35.04)$ & \\
\hline $\begin{array}{l}\text { No. of severe AEs in the past year prior to } \\
\text { the IH }\end{array}$ & & & $<0.001$ \\
\hline 0 & I7I (75.66) & 351 (89.77) & \\
\hline 1 & $26(11.5)$ & $29(7.42)$ & \\
\hline$\geq 2$ & $29(12.83)$ & II (2.8I) & \\
\hline GOLD 2019 classification & & & $<0.001$ \\
\hline Group C & $30(13.27)$ & $114(29.16)$ & \\
\hline Group D & $196(86.73)$ & $277(70.84)$ & \\
\hline \multicolumn{4}{|l|}{ Comorbidity } \\
\hline Cerebrovascular disease and NM disease & $16(7.08)$ & $22(5.63)$ & 0.470 \\
\hline Ischemic heart disease & $25(11.06)$ & $60(15.35)$ & 0.137 \\
\hline Congestive heart failure & $20(8.85)$ & $23(5.88)$ & 0.163 \\
\hline Chronic renal failure stage IV & $8(3.54)$ & $\mathrm{II}(2.8 \mathrm{I})$ & 0.615 \\
\hline Hypertension & 81 (35.84) & $150(38.36)$ & 0.533 \\
\hline Diabetes mellitus & $24(10.62)$ & $54(|3.8|)$ & 0.251 \\
\hline Liver cirrhosis & $4(1.77)$ & $4(1.02)$ & 0.674 \\
\hline Autoimmune disease and cancer & $14(6.19)$ & $38(9.72)$ & 0.129 \\
\hline Charlson index & $4.70 \pm 1.34$ & $4.56 \pm I .43$ & 0.244 \\
\hline
\end{tabular}

Notes: Data are presented as mean \pm SD or $n$ (\%). The significant $\mathrm{P}$ value is shown in bold.

Abbreviations: BMI, body mass index; CRP, C-reactive protein; IH, index hospitalization; NM, neuromuscular disease; AE, acute exacerbation. 
Table 2 Pulmonary Function Test Results and Arterial Blood Gases on Admission (Frequent Exacerbator Group vs NonFrequent Exacerbator Group)

\begin{tabular}{|c|l|l|l|}
\hline Characteristics & $\begin{array}{l}\text { Frequent } \\
\text { Exacerbator } \\
\text { Group (N=226) }\end{array}$ & $\begin{array}{l}\text { Non-Frequent } \\
\text { Exacerbator } \\
\text { Group (N=39I) }\end{array}$ & P value \\
\hline FVC (L) & $2.04 \pm 0.75$ & $2.12 \pm 0.71$ & 0.202 \\
\hline FVC (\% of predict) & $76.12 \pm 27.59$ & $78.14 \pm 23.72$ & 0.357 \\
\hline FEVI (L) & $1.07 \pm 0.46$ & $1.20 \pm 0.47$ & $\mathbf{0 . 0 0 I}$ \\
\hline FEVI (\% of predict) & $51.80 \pm 21.44$ & $56.23 \pm 20.70$ & 0.012 \\
\hline FEVI/FVC \% & $52.63 \pm 12.00$ & $56.45 \pm 11.24$ & $<0.001$ \\
\hline Arterial blood gas & & & $\begin{array}{l}7.38 \pm 0.06 \\
\text { PH value } \\
\text { PaCO }\end{array}$ mmHg \\
\hline
\end{tabular}

Notes: Data are presented as mean $\pm S D$. The significant $P$ value is shown in bold. Abbreviations: FEVI, forced expiratory volume in I second; FVC, forced vital capacity; $\mathrm{PaCO}_{2}$, arterial oxygen and carbon dioxide tensions.

reveal that eosinophilic COPD exacerbation is a wellknown risk factor for readmissionsdue to COPD.

A recent study reported a lower risk in patients with all-cause readmission having blood ECs above 300 cells/ $\mu \mathrm{L} .{ }^{17}$ However, there is conflicting evidence with analyses of two previous cohort studies showing no association between blood eosinophils and increased exacerbation risk. ${ }^{18,19}$ These differences may come for several reasons: 1) The different cutoffs for the definition of human blood eosinophil cell showing inconsistent results associated with the influence of eosinophil cell counts on readmissions throughout sensitivity analyses; 2) The range of respiratory diagnoses included in the intervention study was physician defined. For example, a study recruited the first three ICD coding with COPD diagnosis, and some excluded the diagnosis of pneumonia. ${ }^{17,20}$ In this study, the patients without evidence of previous corroborative spirometry or imaging were excluded. 3) Methodological differences reveal that positive studies were exempt from the confounding effect of systemic corticosteroid use. The primary consideration was the effect of the relative timing of systemic corticosteroid administration on eosinophil level. ${ }^{14}$ 4) Difference of following times after discharge for COPD-related readmission. ${ }^{20}$ One study excluded the patients with $>4$ hospitalizations in the previous 12 months for any cause. ${ }^{21}$

In this study, the FEV1 value and FEV1/FVC ratio were lower in the frequent exacerbator group. Lower FEV1 values were significantly associated with an increased hospital readmission risk. Besides, cases with the FEV1 values of $<50 \%$ showed a substantially lower probability of no readmission during the 1-year follow-up period than cases with $\geq 50 \%$. A previous study indicated that $\mathrm{FEV} 1<50 \%$ was one of the independent predictors of frequent severe exacerbations. ${ }^{22}$ Another study revealed that the requirement for ventilatory support increased the likelihood of readmission by logistic regression analysis of patient-related factors. COPD stage IV patients were reported to have a higher need for ventilatory support. ${ }^{23}$ Poor lung function was related to frequent severe exacerbations as expected, and spirometry data admitting to clinicians would confirm the diagnosis and risk-stratification of patients.

Decreased FEV1 is mainly associated with increased symptoms and heightened inflammatory response, thus may alter an exacerbation risk. ${ }^{24}$ The frequent exacerbations are positively associated with a rapid decline of FEV1 $1{ }^{25}$ Donaldson et $\mathrm{al}^{26}$ reported a mean of 2.92 exacerbations yearly in COPD patients with moderate to very severe disease. The mean rate of FEV1 decline was greater in patients with more exacerbations $(p<0.05)$. Celli et al reported the influence of frequent exacerbations on the FEV1 decline data from the Toward a Revolution in COPD Health (TORCH) study, in which patients experiencing greater frequency of exacerbations had a faster decline in FEV1 during the 3-yr study period..$^{27}$ Repeated episodes of COPD exacerbations might impair lung tissues and lead to an accelerated rate of pulmonary function decline. This concept is supported by patients suffering recurrent exacerbations who showed increased concentrations of inflammatory markers in sputum. ${ }^{28}$ Analysis of exacerbations for 2138 patients enrolled in the ECLIPSE study demonstrated that exacerbations were more frequent and more severe with increased severity of the disease defined using spirometry measures. ${ }^{4}$ Generally, $22 \%$ of patients with stage II disease, 33\% with stage III, and $47 \%$ with stage IV had frequent exacerbations during the first-year follow-up. ${ }^{4}$ The same study also presented that the single best predictor of exacerbations across all GOLD stages was the history of exacerbations. ${ }^{4}$

The readmission rate and risk were higher in patients with two or more severe acute exacerbations in the past year than those with no severe acute exacerbation history in this study. Two previous studies reported that following the first severe COPD exacerbation, each subsequent recurrence requiring hospitalization had increased the risk of a subsequent event and death, with increasing severity of subsequent events. ${ }^{9,29}$ Müllerová et al have 
Table 3 Treatments on Admission and Concurrent Medications (Frequent Exacerbator Group vs Non-Frequent Exacerbator Group)

\begin{tabular}{|c|c|c|c|}
\hline Characteristics & $\begin{array}{l}\text { Frequent Exacerbator Group } \\
(\mathrm{N}=226)\end{array}$ & $\begin{array}{l}\text { Non-Frequent Exacerbator Group } \\
(\mathrm{N}=391)\end{array}$ & $P$ value \\
\hline \multicolumn{4}{|l|}{ Infection status } \\
\hline CXR new patches & $34(15.04)$ & $63(16.11)$ & 0.725 \\
\hline Fever & $25(11.06)$ & $47(12.02)$ & 0.721 \\
\hline Major treatment & & & $0.005 *$ \\
\hline None & $8(3.54)\left(3.57^{*}\right)$ & $8(2.05)(2.07 *)$ & \\
\hline Steroid only & $13(5.75)(5.80 *)$ & $30(7.67)(7.77 *)$ & \\
\hline Antibiotics only & $41(18.14)(18.30 *)$ & II $16(29.67)(30.05 *)$ & \\
\hline Antibiotics+steroid & $162(71.68)(72.32 *)$ & $232(59.34)(60.10 *)$ & \\
\hline Missing & $2(0.88)$ & $5(1.28)$ & \\
\hline ICU admission & $30(13.27)$ & $30(7.67)$ & 0.024 \\
\hline Intubation & $12(5.31)$ & $15(3.84)$ & 0.389 \\
\hline BiPAP use & $20(8.85)$ & $24(6.14)$ & 0.207 \\
\hline RCC admission & $0(0)$ & $2(0.5 I)$ & 0.535 \\
\hline COPD concurrent medications prior to the & & & $<0.001$ \\
\hline \multicolumn{4}{|l|}{$\mathrm{IH}$} \\
\hline None & $80(35.4)$ & $193(49.36)$ & \\
\hline LAMA or LABA & II (4.87) & $27(6.91)$ & \\
\hline LAMA+LABA & $17(7.52)$ & $33(8.44)$ & \\
\hline ICS+LABA & $42(18.58)$ & $64(16.37)$ & \\
\hline LAMA+LABA+ICS & $76(33.63)$ & $74(18.93)$ & \\
\hline COPD concurrent medications following & & & 0.002 \\
\hline \multicolumn{4}{|l|}{ the $\mathrm{IH}$} \\
\hline None & $45(19.91)$ & $115(29.4 I)$ & \\
\hline LAMA or LABA & $10(4.42)$ & $33(8.44)$ & \\
\hline LAMA+LABA & $32(14.16)$ & $68(17.39)$ & \\
\hline ICS+LABA & $44(19.47)$ & $62(15.86)$ & \\
\hline LAMA+LABA+ICS & $95(42.04)$ & $113(28.90)$ & \\
\hline Length of stay, day & $9.46 \pm 7.93$ & $8.14 \pm 8.42$ & 0.055 \\
\hline
\end{tabular}

Notes: Data are presented as mean \pm SD or $\mathrm{n}(\%)$. The significant $\mathrm{P}$ value is shown in bold. *Among patients with available data.

Abbreviations: BiPAP, bilevel positive airway pressure; CXR, Chest X ray; ICS, inhaled corticosteroids; ICU, intensive care unit; IH, index hospitalization; LABA, long-acting beta agonist; LAMA, long-acting muscarinic antagonist; RCC, respiratory care center.

studied a large primary care cohort of 18,568 patients aged $\geq 40$ years in the USA to explore the COPD exacerbation frequency and associated factors. They found noticeable differences between patients managed in primary care who had one or more exacerbations (41.46\%) than patients with no COPD exacerbations recorded during the 12-month follow-up period. $^{23}$ Previous hospitalization for AECOPD was also a strong predictor for future readmission as the rehospitalization frequency is closely linked to the admission frequency in the previous year. ${ }^{30}$ Exacerbation frequency is also increased with raising airflow limitation and similarly with higher dyspnoea score. ${ }^{31}$ The exacerbation frequency was well correlated with symptom severity based on prospective studies' findings, ${ }^{24}$ however, these two factors are semiindependent and recurrent exacerbations could happen in patients with moderate airflow limitation. ${ }^{4}$

In this study, a triple inhaler presented before discharge is one of the independent factors associated with increased severe acute exacerbations risk of COPD. On the contrary, after discharge, a triple inhaler is one of the independent factors for less frequent severe acute exacerbation. Inhaled corticosteroids (ICS) are commonly prescribed to COPD patients to reduce exacerbations. A recent meta-analysis with thirteen randomized controlled trials containing 15,519 patients with COPD (ICS/LABA/LAMA combination, 
A

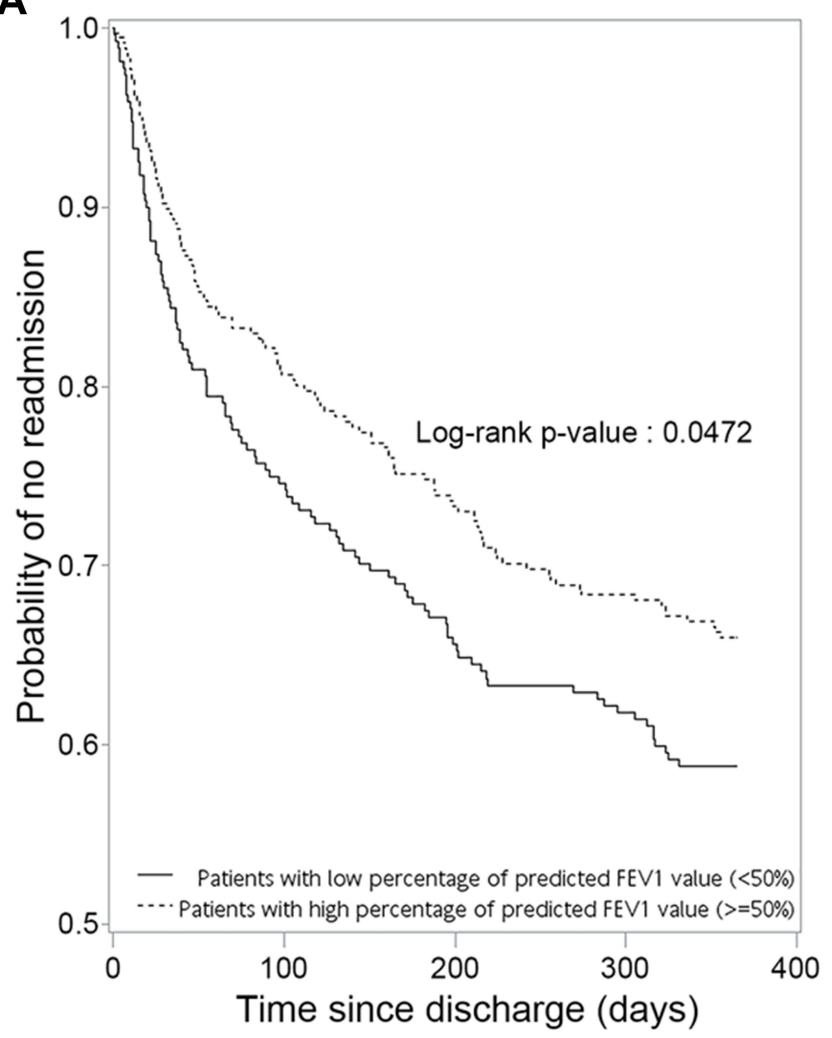

B

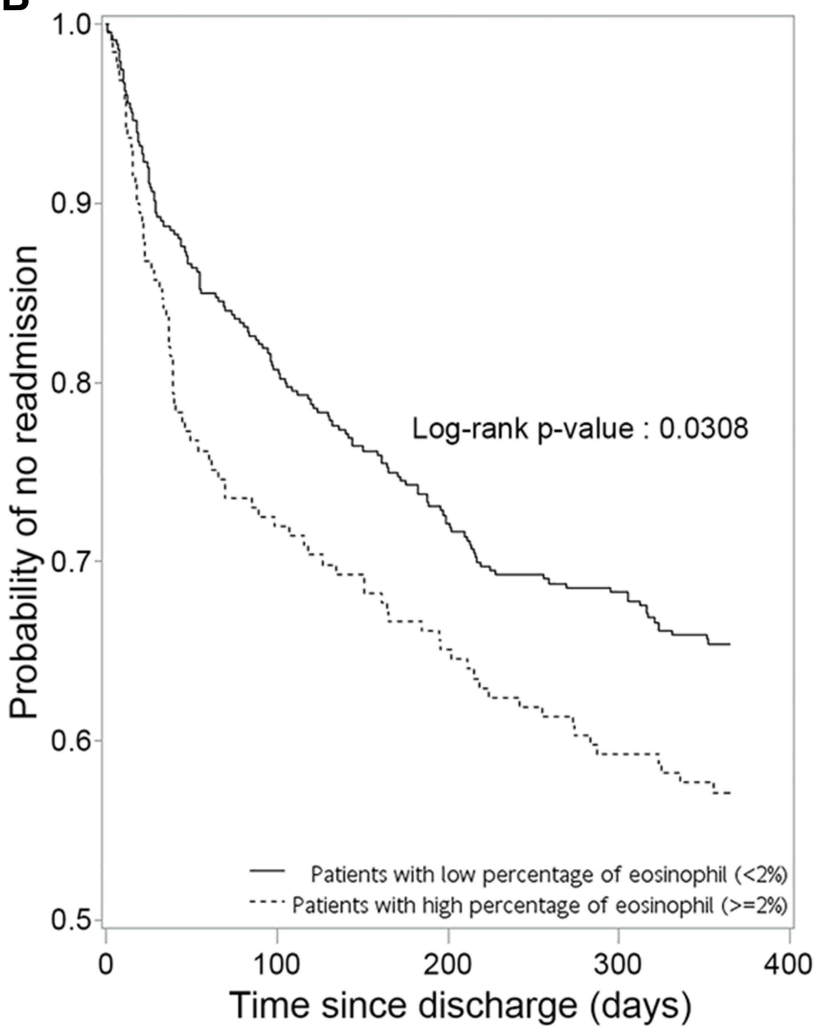

Figure 2 Kaplan-Meier curves for time to hospital readmissions within I year after hospital discharge. (A) Comparison between patients with FEVI <50\% (\% of predicted) and $\mathrm{FEVI} \geq 50 \%$ (\% of predicted). (B) Comparison between patients with eosinophil count $<2 \%$ and eosinophil count $\geq 2 \%$.

53.1\%; ICS/LABA combination, 46.9\%) indicated that combination therapy with ICS/LABA/LAMA was correlated with the more reduction of moderate or severe AECOPD risk (relative risk of $0.78 ; 95 \% \mathrm{CI}, 0.71-0.85$ ) and better lung function than either LABA/LAMA combination therapy or single long-acting bronchodilator therapy. ${ }^{32}$ Besides, compared to LABA/LAMA combination therapy, the ICS/ LABA/LAMA combination therapy was correlated with higher blood ECs, and no significant differences were detected concerning the pneumonia risk between triple combination therapy and comparators. ${ }^{32}$ These findings appear to support the current recommendations of the management strategy for the COPD from Global Initiative for Chronic Obstructive Lung Disease (GOLD), which suggests the use of triple therapy in patients with clinically significant symptoms and are at increased risk for frequent or severe exacerbations (GOLD group D). ${ }^{2}$ Furthermore, the triple therapy showed the same cardiovascular safety profile compared to the ICS/LABA combination. ${ }^{33-37}$

There are several limitations to this study. First, this was a single-center study in Taiwan involving only the Asian race with a relatively small sample size, which might potentially limit the geographical scope of the findings and be affected by the biases related to such studies. Secondly, due to the lack of complete records of some patients, the estimation of exacerbation patients with no hospitalization was absent in this study. Therefore, it might underestimate the frequency of moderate-to-severe exacerbations and lead to analysis bias. Thirdly, discharged patients had to be alive to be included in this study. This might influence intra-hospitalized characteristics such as length of stay, ICU utilization rate, and mechanical ventilation. Thus, no conclusions can be obtained from these data for outcomes during the $\mathrm{IH}$. All analyses were based on a single measurement at the time of $\mathrm{IH}$, and any changes due to treatment or simple disease progression during the in-hospital stay were not assessed. Fourthly, this study considers the time effect of systemic corticosteroid administration and ECs. This possible bias must be recognized that excluding such patients might lose patients with the experience of outpatient treatment failure within the last 48 hours. Finally, although a spirometry recording was required for COPD patients' eligibility in the present study, all COPD patients were not ensured to be enrolled due to lack of PFT. 
Table 4 Hazard Ratios and Event Rates for COPD-Related Readmission in Different Clinical Characteristic Comparisons

\begin{tabular}{|c|c|c|c|c|c|}
\hline Variable & No. & Events & $\begin{array}{l}\text { Event Rate (/100 Person- } \\
\text { Years) }\end{array}$ & $\begin{array}{l}\text { Adjusted } H^{a}(95 \% \\
C l)\end{array}$ & P value \\
\hline Age & 617 & 230 & $|8.5|$ & $1.02(1.00-1.03)$ & 0.025 \\
\hline Eosinophil count (\%) & 617 & 230 & $18.5 \mid$ & $1.09(1.05-1.13)$ & $<0.001$ \\
\hline FEVI (L) & 617 & 230 & $|8.5|$ & $0.72(0.52-0.99)$ & 0.041 \\
\hline \multicolumn{6}{|l|}{ No. of severe AEs in the past year prior to the IH } \\
\hline 0 & 522 & 174 & 16.29 & Reference & \\
\hline I & 55 & 26 & 20.35 & $1.36(0.89-2.09)$ & 0.153 \\
\hline$\geq 2$ & 40 & 30 & 63.90 & $3.38(2.23-5.11)$ & $<0.001$ \\
\hline \multicolumn{6}{|l|}{ COPD concurrent medications prior to the $\mathrm{IH}$} \\
\hline None & 273 & 81 & $13.3 \mid$ & Reference & \\
\hline LAMA or LABA & 38 & 12 & 13.98 & $0.97(0.52-1.80)$ & 0.923 \\
\hline LAMA+LABA & 50 & 17 & 28.38 & $1.22(0.72-2.09)$ & 0.459 \\
\hline$I C S+L A B A$ & 106 & 43 & 18.44 & I.22 (0.84-I.79) & 0.299 \\
\hline$L A M A+L A B A+I C S$ & 150 & 77 & 30.18 & I.83 (1.33-2.54) & $<0.001$ \\
\hline \multicolumn{6}{|l|}{ Smoking status } \\
\hline Never-smoker & 252 & 84 & 15.62 & Reference & \\
\hline Current smoker & 145 & 60 & 23.40 & $1.43(1.01-2.03)$ & 0.044 \\
\hline Ex-smoker & 220 & 86 & 19.19 & $1.05(0.78-1.43)$ & 0.731 \\
\hline
\end{tabular}

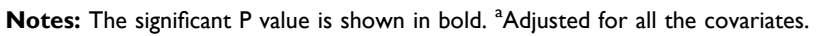

Abbreviations: FEVI, forced expiratory volume in I second; AE, acute exacerbation; IH, index hospitalization; LABA, long-acting beta agonist; LAMA, long-acting muscarinic antagonist; ICS, inhaled corticosteroids.

\section{Conclusion}

The distinct COPD exacerbation subtype of severe acute exacerbations was proposed and showed a difference in prognosis and treatment response. EC $\geq 2 \%$, FEV1 $<50 \%$, and patients with severe acute exacerbations history of at least 2 in 1 year of postexacerbation before the IH might be the predictors of the frequent exacerbator COPD phenotype. Besides, higher age, triple combination inhaler before the IH, and smoking might be independently correlated with the frequent readmission risk within 1-year postexacerbation. These findings underline the importance of identifying COPD patients with a risk of frequent exacerbation, and the management of COPD should involve phenotype-directed strategies. In addition, these results provide information for physicians to a better understanding of the mechanisms of COPD exacerbations with more prospective studies.

\section{Ethics Approval and Informed Consent}

The study was approved by the Institutional Review Board, Taipei Tzu Chi Hospital, Buddhist Tzu Chi
Medical Foundation in August 2020 (protocol number 09-X-059). Written informed consent was signed and provided by each included participant.

\section{Author Contributions}

All authors made substantial contributions to conception and design, acquisition of data, or analysis and interpretation of data; took part in drafting the article or revising it critically for important intellectual content; agreed to submit to the current journal; gave final approval for the version to be published; and agreed to be accountable for all aspects of the work.

\section{Funding}

There is no funding to report.

\section{Disclosure}

The authors report no conflicts of interest in this work.

\section{References}

1. Singh D, Agusti A, Anzueto A, et al. Global Strategy for the Diagnosis, Management, and Prevention of Chronic Obstructive Lung Disease: the GOLD science committee report 2019. Eur Respir J. 2019;53(5):1900164. Published 2019 May 18. doi:10.1183/ 13993003.00164-2019 
2. Vogelmeier CF, Criner GJ, Martinez FJ, et al. Global strategy for the diagnosis, management, and prevention of chronic obstructive lung disease 2017 report: GOLD executive summary. Eur Respir J. 2017;49:557-582. doi:10.1183/13993003.00214-2017

3. Le Rouzic O, Roche N, Cortot AB, et al. Defining the "frequent exacerbator" phenotype in COPD: a hypothesis-free approach. Chest. 2018;153:1106-1115. doi:10.1016/j.chest.2017.10.009

4. Hurst JR, Vestbo J, Anzueto A, et al. Susceptibility to exacerbation in chronic obstructive pulmonary disease. $N$ Engl $J$ Med. 2010;363:1128-1138. doi:10.1056/NEJMoa0909883

5. Vestbo J, Edwards LD, Scanlon PD, et al. Changes in forced expiratory volume in 1 second over time in COPD. $N$ Engl $J$ Med. 2011;365:1184-1192. doi:10.1056/NEJMoa1105482

6. Andersson F, Borg S, Jansson SA, et al. The costs of exacerbations in chronic obstructive pulmonary disease (COPD). Respir Med. 2002;96:700-708. doi:10.1053/rmed.2002.1334

7. Iacobucci G. Hospital readmissions for COPD in England are rising, audit shows. BMJ. 2017;356:j557. doi:10.1136/bmj.j557

8. Press VG, Au DH, Bourbeau J, et al. Reducing chronic obstructive pulmonary disease hospital readmissions. An official American Thoracic Society workshop report. Ann Am Thorac Soc. 2019;16:161-170. doi:10.1513/AnnalsATS.201811-755WS

9. Pavord ID, Jones PW, Burgel PR, Rabe KF. Exacerbations of COPD. Int J Chron Obstruct Pulmon Dis. 2016;11(Spec Iss):21-30.

10. McGarvey L, Lee AJ, Roberts J, et al. Characterisation of the frequent exacerbator phenotype in COPD patients in a large UK primary care population. Respir Med. 2015;109:228-237. doi:10.1016/j. rmed.2014.12.006

11. Bélanger M, Couillard S, Courteau J, et al. Eosinophil counts in first COPD hospitalizations: a comparison of health service utilization. Int J Chron Obstruct Pulmon Dis. 2018;13:3045-3054. doi:10.2147/ COPD.S170743

12. Couillard S, Larivée P, Courteau J, Vanasse A. Eosinophils in COPD exacerbations are associated with increased readmissions. Chest. 2017;151:366-373. doi:10.1016/j.chest.2016.10.003

13. Wu CW, Lan CC, Hsieh PC, Tzeng IS, Wu YK. Role of peripheral eosinophilia in acute exacerbation of chronic obstructive pulmonary disease. World J Clin Cases. 2020;8:2727-2737. doi:10.12998/wjcc.v8.i13.2727

14. Li Q, Larivée P, Courteau J, et al. Greater eosinophil counts at first COPD hospitalization are associated with more readmissions and fewer deaths. Int J Chron Obstruct Pulmon Dis. 2019;14:331-341. doi:10.2147/COPD.S187375

15. Vedel-Krogh S, Nielsen SF, Lange P, Vestbo J, Nordestgaard BG. Blood eosinophils and exacerbations in chronic obstructive pulmonary disease. The Copenhagen General Population Study. Am J Respir Crit Care Med. 2016;193:965-974. doi:10.1164/rccm.201509-1869OC

16. Yun JH, Lamb A, Chase R, et al. Blood eosinophil count thresholds and exacerbations in patients with chronic obstructive pulmonary disease. J Allergy Clin Immunol. 2018;141:2037-2047 e2010. doi:10.1016/j.jaci.2018.04.010

17. Håkansson KEJ, Ulrik CS, Godtfredsen NS, et al. High suPAR and low blood eosinophil count are risk factors for hospital readmission and mortality in patients with COPD. Int J Chron Obstruct Pulmon Dis. 2020;15:733-743.

18. Casanova C, Celli BR, de-Torres JP, et al. Prevalence of persistent blood eosinophilia: relation to outcomes in patients with COPD. Eur Respir J. 2017;50(5):1701162. Published 2017 Nov 22. doi:10.1183/ 13993003.01162-2017

19. Hastie AT, Martinez FJ, Curtis JL, et al. Association of sputum and blood eosinophil concentrations with clinical measures of COPD severity: an analysis of the SPIROMICS cohort. Lancet Respir Med. 2017;5:956-967. doi:10.1016/S2213-2600(17)30432-0

20. Duman D, Aksoy E, Agca MC, et al. The utility of inflammatory markers to predict readmissions and mortality in COPD cases with or without eosinophilia. Int $J$ Chron Obstruct Pulmon Dis. 2015;10:2469-2478. doi:10.2147/COPD.S90330
21. Bafadhel M, Greening NJ, Harvey-Dunstan TC, et al. Blood eosinophils and outcomes in severe hospitalized exacerbations of COPD. Chest. 2016;150:320-328. doi:10.1016/j.chest.2016.01.026

22. Ozyilmaz E, Kokturk N, Teksut G, Tatlicioglu T. Unsuspected risk factors of frequent exacerbations requiring hospital admission in chronic obstructive pulmonary disease. Int $J$ Clin Pract. 2013;67:691-697. doi:10.1111/ijcp.12150

23. Hartl S, Lopez-Campos JL, Pozo-Rodriguez F, et al. Risk of death and readmission of hospital-admitted COPD exacerbations: European COPD audit. Eur Respir J. 2016;47:113-121.

24. Donaldson GC, Wedzicha JA. COPD exacerbations.1: epidemiology. Thorax. 2006;61:164-168. doi:10.1136/thx.2005.041806

25. Anzueto A, Leimer I, Kesten S. Impact of frequency of COPD exacerbations on pulmonary function, health status and clinical outcomes. Int J Chron Obstruct Pulmon Dis. 2009;4:245-251.

26. Donaldson GC, Seemungal TA, Bhowmik A, Wedzicha JA. Relationship between exacerbation frequency and lung function decline in chronic obstructive pulmonary disease. Thorax. 2002;57:847-852. doi:10.1136/thorax.57.10.847

27. Celli BR, Thomas NE, Anderson JA, et al. Effect of pharmacotherapy on rate of decline of lung function in chronic obstructive pulmonary disease: results from the TORCH study. Am J Respir Crit Care Med. 2008;178:332-338. doi:10.1164/rccm.200712-1869OC

28. Crooks SW, Bayley DL, Hill SL, Stockley RA. Bronchial inflammation in acute bacterial exacerbations of chronic bronchitis: the role of leukotriene B4. Eur Respir J. 2000;15:274-280. doi:10.1034/j.13993003.2000.15b09.x

29. Suissa S, Dell'Aniello S, Ernst P. Long-term natural history of chronic obstructive pulmonary disease: severe exacerbations and mortality. Thorax. 2012;67:957-963. doi:10.1136/thoraxjnl-2011201518

30. Baker CL, Zou KH, Su J. Risk assessment of readmissions following an initial COPD-related hospitalization. Int J Chron Obstruct Pulmon Dis. 2013;8:551-559.

31. Müllerová H, Shukla A, Hawkins A, Quint J. Risk factors for acute exacerbations of COPD in a primary care population: a retrospective observational cohort study. BMJ Open. 2014;4:e006171.

32. Cazzola M, Rogliani P, Calzetta L, Matera MG. Triple therapy versus single and dual long-acting bronchodilator therapy in COPD: a systematic review and meta-analysis. Eur Respir J. 2018;52 (6):1801586. doi:10.1183/13993003.01586-2018

33. Calzetta L, Cazzola M, Matera MG, Rogliani P. Adding a LAMA to ICS/LABA therapy: a meta-analysis of triple combination therapy in COPD. Chest. 2019;155(4):758-770. doi:10.1016/j.chest.2018.12.016

34. Ferguson GT, Rabe KF, Martinez FJ, et al. Triple therapy with budesonide/glycopyrrolate/formoterol fumarate with co-suspension delivery technology versus dual therapies in chronic obstructive pulmonary disease (KRONOS): a double-blind, parallel-group, multicentre, phase 3 randomised controlled trial. Lancet Respir Med. 2018;6:747-758.

35. Lipson DA, Barnacle H, Birk R, et al. FULFIL trial: once-daily triple therapy for patients with chronic obstructive pulmonary disease. $\mathrm{Am}$ $J$ Respir Crit Care Med. 2017;196:438-446. doi:10.1164/rccm.20 1703-0449OC

36. Lipson DA, Barnhart F, Brealey N, et al. Once-daily single-inhaler triple versus dual therapy in patients with COPD. $N$ Engl J Med. 2018;378:1671-1680. doi:10.1056/NEJMoa1713901

37. Singh D, Papi A, Corradi M, et al. Single inhaler triple therapy versus inhaled corticosteroid plus long-acting $\beta 2$-agonist therapy for chronic obstructive pulmonary disease (TRILOGY): a double-blind, parallel group, randomised controlled trial. Lancet. 2016;388:963-973. doi:10.1016/S0140-6736(16)31354-X 


\section{Publish your work in this journal}

The International Journal of COPD is an international, peer-reviewed journal of therapeutics and pharmacology focusing on concise rapid reporting of clinical studies and reviews in COPD. Special focus is given to the pathophysiological processes underlying the disease, intervention programs, patient focused education, and self management

protocols. This journal is indexed on PubMed Central, MedLine and CAS. The manuscript management system is completely online and includes a very quick and fair peer-review system, which is all easy to use. Visit http://www.dovepress.com/testimonials.php to read real quotes from published authors.

Submit your manuscript here: https://www.dovepress.com/international-journal-of-chronic-obstructive-pulmonary-disease-journal 